\title{
Using Cellular Automata to Investigate Pedestrian Conflicts with Vehicles in Crosswalk at Signalized Intersection
}

\author{
Xiaomeng Li, Xuedong Yan, Xingang $L i$, and Jiangfeng Wang \\ MOE Key Laboratory for Urban Transportation Complex Systems Theory and Technology, \\ Beijing Jiaotong University, Beijing 100044, China \\ Correspondence should be addressed to Xuedong Yan, xdyan@bjtu.edu.cn
}

Received 1 August 2012; Accepted 18 October 2012

Academic Editor: Bo Yang

Copyright (C) 2012 Xiaomeng Li et al. This is an open access article distributed under the Creative Commons Attribution License, which permits unrestricted use, distribution, and reproduction in any medium, provided the original work is properly cited.

\begin{abstract}
The operational efficiency and safety of pedestrian flows at intersections is an important aspect of urban traffic. Particularly, conflicts between pedestrians and vehicles in crosswalk are one of the most influential factors for intersection safety. This paper presents a cellular automata model that simulates pedestrian and vehicle crossing behaviors at signalized intersections. Through the simulation, we investigate the effects of different pedestrian signal timing and crosswalk widths on the crosswalk capacity, the number of traffic conflicts between pedestrians and vehicles, and pedestrian delay due to the conflicts. The simulation results indicate that the cellular automata is an effective simulation platform for investigating complex pedestrian-related traffic phenomenon at signalized intersections.
\end{abstract}

\section{Introduction}

Intersections are the basic joints of urban roads, through which multidirection traffic flows pass. Thus, traffic conflicts and crashes are prone to happen at the intersections. In China, the mixed traffic flow is one of the typical characteristics of urban road traffic. Pedestrians account for a great proportion in the intersection traffic and they are vulnerable road users who have to face long crossing distance that increases exposure to conflicts with intersecting vehicles. Even worse, the time for pedestrians to finish their crossing is often insufficient due to the large intersection size and heavy pedestrian traffic demand. So, the potential conflicts between pedestrians and vehicles may lead to traffic crashes, resulting in injuries and fatalities more likely for pedestrians. According to the statistics, about one-third of the road traffic crashes in China are directly associated with pedestrians and about $25 \%$ 
of the accident death tolls are from pedestrian traffic [1]. Besides, the traffic conflicts can also cause delay problem, which decreases the efficiency of road traffic to a certain extent. Therefore, it is practically meaningful to study the conflicts between pedestrians and vehicles at intersections.

Better understanding the generation mechanism of traffic conflict at intersections can contribute to investigating the causation of traffic crashes. Perkins and Harris [2] first defined traffic conflict as "an interaction between two or more road users and an incident that induces the avoidance behavior of road users to avoid an imminent accident." A formalized definition of traffic conflict was later adopted as "an observable situation in which two or more road users approach each other in space and time for such an extent that there is a risk of collision if their movements remain unchanged" [3]. The methods for traffic conflict research can be mainly classified into three categories, including excavation and analysis of measured data [4-7], modeling analysis of conflict mechanism [8-10], and simulation-based analysis, which tends to apply traffic simulation software [11] or develop traffic simulation model [12]. Compared with the others, the simulation method is often used in practice as an alternative analysis tool and more flexible and promising to overcome the limitations of existing procedures [13].

The previous studies related to traffic conflicts in China and other countries mainly involve the following aspects: (1) recognition of traffic conflict and how to define its severity [14-16]; (2) prediction of traffic conflict and its relationship with traffic accident [17-19]; (3) application of traffic conflict technology in safety evaluation [20-23]. However, most of the studies about road traffic conflicts focus on conflicts between vehicles, or the interaction between pedestrians and vehicles with mathematical formula models [24], and few researchers pay attention to conflicts related to pedestrians with microscopic simulation model, such as cellular automata model.

In this paper, we choose the cellular automata as a simulation model to analyze the crossing behavior of pedestrians and their conflicts with vehicles in crosswalks at signalized intersections. Because the cellular automata method has advantages in characterizing the traffic flow's discreteness features and easy computer simulation, it has been used to study traffic flow simulation by many scholars [25-31]. In recent years, the cellular automata has been applied to investigate pedestrian movements and behavior [32-35]. Compared to vehicle, the simulation of pedestrian is more challenging. In many aspects, pedestrians' movements are more complicated and random. However, the cellular automata simulation method can deal with the complexity and uncertainty of pedestrian movement process through properly describing traffic behavior rules rather than creating mathematical models. This simulation study based on the cellular automata method aims to (1) quantitatively evaluate crosswalk capacity, the number of traffic conflicts between pedestrians and vehicles, and pedestrian delay due to the conflicts at a signalized intersection and (2) explore how they are associated with pedestrian signal timing and crosswalk width.

\section{Cellular Automata Model}

The cellular automata is idealization of physical system in which both space and time are assumed to be discrete and each of the interacting units can have only a finite number of discrete states [36]. In the cellular automata, the space is divided into many units by inerratic grid with certain forms. Every unit in the inerratic grid is called a cell, which can only take its value in the limited discrete state set. All the cells follow the same function rules and 
update according to the fixed partial rules. A large number of cells constitute the evolution of the dynamic system through the simple interaction. The most basic components of cellular automata include four parts: cells, cell space, neighbors, and rules [37]. Besides, the state of cells should also be included. The concept of cellular automata was first introduced in the 1950s by von Neumann while formulating an abstract theory of self-replicating computing machines [38]. However, it received the attention of a wider audience in the 1970s through Conway's game of life [39]. The first CA model for vehicular traffic was introduced by Cremer and Ludwig [40]. After development and transformation of decades years, the cellular automata approach proved to be quite useful, not only in the field of vehicular traffic flow modeling, but also in other fields such as pedestrian behavior, escape and panic dynamics, the spreading of forest fires, population growth and migrations, cloud formation, material properties (corrosion, cracks, creases, peeling, etc.), ant colonies, and pheromone trails [41-45].

The cellular automata model in this paper is developed to simulate a crosswalk at a signalized intersection with a fixed pedestrian signal time cycle of $90 \mathrm{~s}$. The phase of pedestrian signal is designed as six classes, which are $25 \mathrm{~s}, 30 \mathrm{~s}, 35 \mathrm{~s}, 40 \mathrm{~s}, 45 \mathrm{~s}$, and $50 \mathrm{~s}$ green time length, corresponding to $65 \mathrm{~s}, 60 \mathrm{~s}, 55 \mathrm{~s}, 50 \mathrm{~s}, 45 \mathrm{~s}$, and $40 \mathrm{~s}$ red time length, respectively. The road segment connecting to the intersection is designed as two-way six-vehicle lanes and each lane's width is 3.5 meters. The crosswalk's width is designed into 6 classes too, which are $2.5 \mathrm{~m}, 3 \mathrm{~m}, 3.5 \mathrm{~m}, 4 \mathrm{~m}, 4.5 \mathrm{~m}$ and $5 \mathrm{~m}$ with a $21 \mathrm{~m}$ crossing length. In addition, two pedestrian waiting areas are set on both sides of the crosswalk which can hold 100 pedestrians at most each time.

In this model, the crosswalk is divided into $0.5 \times 0.5 \mathrm{~m}^{2}$ square cells. Each cell is either occupied by one pedestrian or empty. The initial assigned velocities of the pedestrian can be $2,3,4,5$, or 6 cells per second, which represents that their actual velocities are $1 \mathrm{~m} / \mathrm{s}, 1.5 \mathrm{~m} / \mathrm{s}$, $2 \mathrm{~m} / \mathrm{s}, 2.5 \mathrm{~m} / \mathrm{s}$, and $3 \mathrm{~m} / \mathrm{s}$, respectively. The ratios of the five kinds of velocities are based on the field observation results, which share $27.3 \%, 52 \%, 13.7 \%, 4.8 \%$ and $2.2 \%$ correspondingly. Through comparison of the assigned velocity and the number of vertically front empty cells, each pedestrian updates his/her velocity at each time step. Each vehicle is assumed to occupy $6 \times 5$ cells and its velocity at the crosswalk is $5 \mathrm{~m} / \mathrm{s}$.

There are usually two kinds of boundary conditions in cellular automata simulation models: the periodic boundary condition and the open boundary condition [36]. For both practical and theoretical reasons, the two boundary conditions are preferable in different situations. In the periodic boundary condition, the relative boundaries are linked and a translation invariant stationary state is generated in the system. On the other hand, the open boundary condition accords with a certain probability when a vehicle is inserted and removed from the boundary cell, in which the translational invariance is broken but one can still expect stationary states with a nontrivial density profile. Compared with the periodic boundary condition, the open boundary condition is more realistic and consilient for the model in this paper. Thus, the open boundary condition is adopted.

\section{Cellular Automata Evolution Rules}

There are three basic laws of motion in cellular automata models to describe the behavior of pedestrians: lateral movement, linear movement, and collision avoidance [46]. The lateral movement represents a pedestrian's path change behavior, the result of which is to obtain acceleration space and avoid a head-on collision. The linear movement needs to considerate 


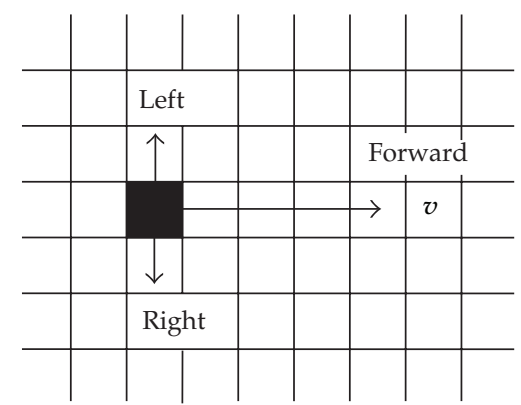

Figure 1: The pedestrian movement neighborhood.

the personal preference speed and other nearby pedestrians to identify the number of steps for forward movement. The collision avoidance refers to behavior of how to avoid head-on collisions for pedestrians walking close to each other along the opposite direction. In the evolution of this model, both pedestrians and vehicles have to comply with the rules of their movements to perform the lateral movement, linear movement, and collision avoidance.

\subsection{Rules for Pedestrian}

(1) The arrival rate of pedestrian flow obeys the Poisson distribution as shown in (3.1)

$$
P_{k}=\frac{\left(\lambda_{p} t\right)^{k}}{k !} e^{-\lambda t}
$$

where, $P_{k}$ denotes the probability of reaching $k$ pedestrians during the counting interval $t$; $\lambda_{p}$ denotes the average pedestrian arrival rate (capita/second); $t$ denotes every time interval counted.

(2) In the evolution of this model, pedestrians give priority to the front cell as their destination. Pedestrian can move forward $v$ cells or change to the left or the right cell when he/she is blocked (see Figure 1).

(a) Step forward: the initial velocity of pedestrian going into the crosswalk is assigned in (3.2) according to field observation:

$$
v_{i, j}= \begin{cases}6 & \text { if } p>0.978 \\ 5 & \text { if } 0.978 \geq p>0.93 \\ 4 & \text { if } 0.93 \geq p>0.793 \\ 3 & \text { if } 0.793 \geq p>0.273 \\ 2 & \text { if } 0.273 \geq p \geq 0\end{cases}
$$

where, $p$ is a random number between 0 and $1 ; v_{i, j}$ denotes the velocity of pedestrian whose location is $x_{i, j}$. 

(3.3):

The velocity of pedestrian in crosswalk during simulation is updated on the basis of

$$
v_{i, j}=\min \left\{d_{i, j}, v_{i, j}\right\}
$$

where, $d_{i, j}$ denotes the number of empty cells between the target pedestrian and his/her nearest vertically front pedestrian.

(b) Lane change: if any condition in (3.4), (3.5), or (3.6) is satisfied, lane change happens. If the condition in (3.4) is satisfied, the pedestrian will change to the left or the right cell with the same probability. If the condition in (3.5) is satisfied, the pedestrian will change to the right cell. If the condition in (3.6) is satisfied, he/she will change to the left cell:

$$
\begin{gathered}
d_{i, j}=0 \& \& x_{i, j+1}=x_{i, j-1}=0 \& \& d_{i, j \pm 1}>v_{i, j} \& \& v_{i-n, j \pm 1}<v_{i, j}, \\
d_{i, j}=0 \& \& x_{i, j+1}=0 \& \& x_{i, j-1}=1 \& \& d_{i, j+1}>v_{i, j} \& \& v_{i-n, j+1}<v_{i, j}, \\
d_{i, j}=0 \& \& x_{i, j-1}=0 \& \& x_{i, j+1}=1 \& \& d_{i, j-1}>v_{i, j} \& \& v_{i-n, j-1}<v_{i, j},
\end{gathered}
$$

where, $x_{i, j}$ denotes the location of pedestrian; $v_{i-n, j \pm 1}$ denotes the speed of pedestrian whose location is $x_{i-n, j \pm 1}$ and it's the first one behind the cell $x_{i, j \pm 1}$. The number of empty cells between them is $n$.

(3) For the reason that several pedestrians might compete for one empty cell and each cell can either be empty or occupied by one pedestrian exactly, conflicts will exist among pedestrians in the evolution of the model. If conflicts happen, the system will select one pedestrian to occupy the cell randomly with an equal probability. The selected pedestrian will move to the destination at the next time-step and the pedestrian who is not selected will stay at his/her original location.

(4) The pedestrians have to wait at the waiting area once they arrive at the red light time and all of the pedestrians are assumed to follow the traffic rules with no intentional red light running behavior. The red light running behavior only happens on the pedestrians that have not finished crossing when the green light ends. When the green light begins, they will start crossing at the assigned velocities and accelerate to the maximum velocity to complete crossing if the green light ends.

(5) The model simplifies the conflict process between opposite pedestrians. The opposite-direction pedestrians can span each other if their destination cell is empty; otherwise, they need to adjust their velocities or keep original position.

\subsection{Rules for Vehicle}

(1) The arrival law of vehicles is the same as pedestrians, which follows the Poisson distribution similarly. Its arrival rate is $\lambda_{v}$.

(2) When the pedestrian light is red and there is no pedestrian on the crosswalk, the vehicles can cross through the intersection. Otherwise, they would encounter the conflicts with pedestrians. 


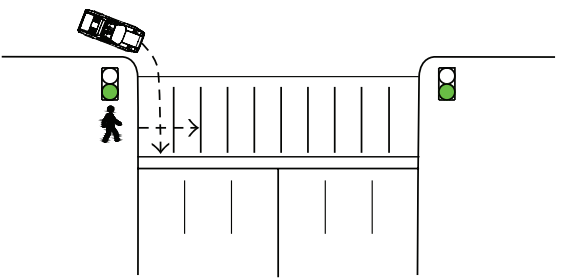

(a) Conflict area 1

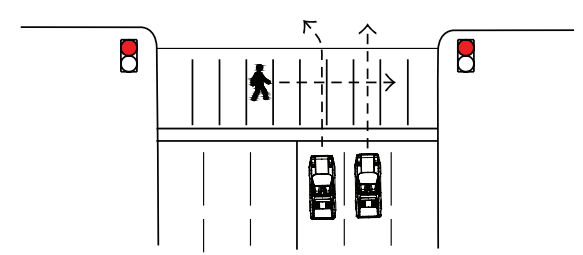

(c) Conflict area 3

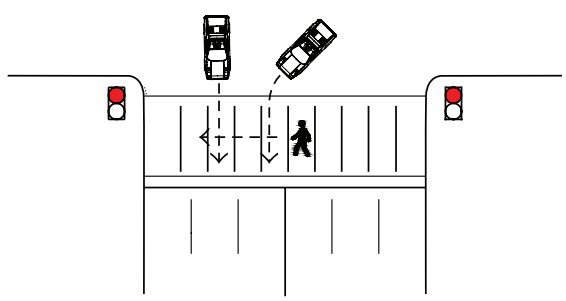

(b) Conflict area 2

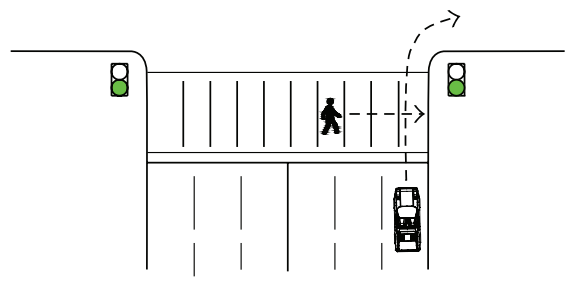

(d) Conflict area 4

Figure 2: Conflict areas.

\subsection{Rules for Conflict between Pedestrian and Vehicle}

\subsubsection{Conflict Area}

In this paper, according to the time and space occupation relationship of road users at the intersection, the conflicts between pedestrians and vehicles are classified as 4 areas, as shown in Figure 2, in which the conflicts between pedestrians and vehicles can be differentiated into 4 types as follows.

Area 1: conflicts between right-turning vehicles from inside of intersection and pedestrians (see Figure 2(a)), assuming that right turn on red is allowed at the approach.

Area 2: conflicts between straight-moving or left-turning vehicles from inside of intersection and pedestrians with slow speed or those who cross the road at the end of green light (see Figure 2(b)), assuming that the vehicles have no red light running behavior.

Area 3: conflicts between straight-moving or left-turning vehicles from the segment and pedestrians with slow speed or those who cross the road at the end of green light (see Figure 2(c)), assuming that the vehicles have no red-light running behavior.

Area 4: conflicts between right-turning vehicles from the segment and pedestrians (see Figure 2(d)), assuming that right turn on red is allowed at the approach.

\subsubsection{Conflict-Related Definitions and Conflict Rules}

There are two conflict-related definitions involved in this paper. One is the conflict event between pedestrian and vehicle, and the other is the pedestrian conflict delay. In this simulation study, a conflict event between vehicle and pedestrian in crosswalk is defined 
as the situation that in order to avoid a collision with pedestrian, the vehicle stops moving forward into the cells which are currently occupied by at least one pedestrian or will be occupied by at least one pedestrian. The pedestrian conflict delay means the stop time during which the pedestrians stagnate in crosswalk to avoid passing-through vehicles when a conflict happens. If the conflict is relieved, pedestrians can continue to cross. The conflict rules are assigned as follows.

(1) If the pedestrian's target cell is occupied by a vehicle, the vehicle has the priority to cross through. In the same way, if the vehicle's target cell is occupied by pedestrians, the priority will be given to the pedestrians.

(2) If the pedestrian and the vehicle have the same target cell, which means their next time steps overlap, the system will give the priority of passage to one of them randomly with an equal probability, namely, 0.5 .

\section{Simulation Results}

The model uses MATLAB as the simulation platform. The span for each simulation time step is set as 1 second. The simulation data is recorded every 3600 time steps, which is equal to one hour. For each simulation scenario, thirty-round simulations were conducted and the average values of the thirty simulation observations are used for result analyses.

\subsection{Crosswalk Traffic Capacity}

The traffic capacity of crosswalk is a basic parameter for pedestrian crossing design. If the capacity cannot meet the pedestrian traffic demand, it will affect the pedestrian crossing efficiency and increase the possibility of conflict between pedestrian and vehicle, which might lead to potential traffic crashes. In this paper, the pedestrian green light time and the crosswalk width's effect on the crosswalk capacity are studied. Figure 3 shows that, when the pedestrian signal timing and the width of the pedestrian crosswalk are given, the number of pedestrians that complete crossing during green light increases with the increase of pedestrian flow first and then decreases. As the increment of pedestrian flow, the resistance between opposing pedestrian flows becomes larger, which makes the pedestrian velocity slow significantly. Thus the number of pedestrians completing crossing during green time decreases. Additionally, when the pedestrian traffic flow is lower, the vehicle flow does not have a significant effect on pedestrian flow since fewer conflicts between pedestrians and vehicles occur. Once the pedestrian flow reaches a certain degree (above $2000 \mathrm{cap} / \mathrm{hr}$ ), the larger the vehicle flow is, the less the pedestrians finish crossing during the green light. The maximum number of the pedestrians that complete crossing during the same green phase is defined as the crosswalk traffic capacity for the specific signal timing and crosswalk width.

Figure 4 shows the regression results between green time and capacity using a quadratic model for the six types of cross widths (CW), which give superb goodness-of-fit measures $\left(R^{2}>0.97\right)$. Based on the simulation analysis, it can be found that if the pedestrian cycle timing is available, the pedestrian traffic capacity can be calculated by green length through the fitted formulas. 


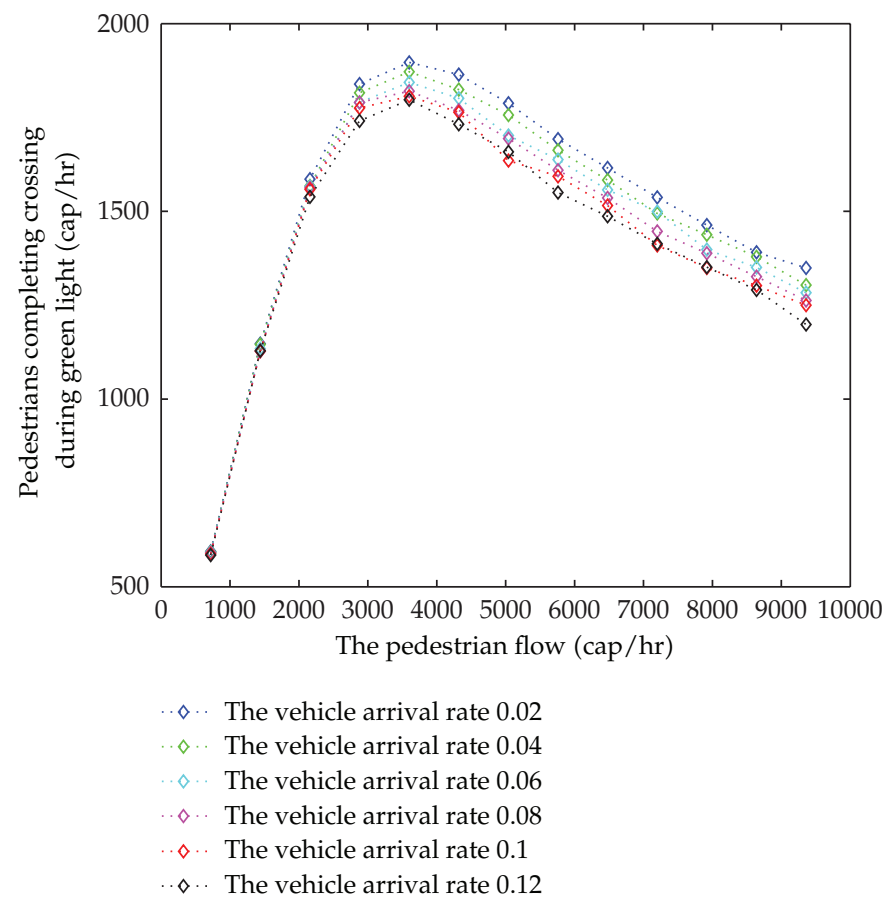

Figure 3: The relationship between pedestrians completing crossing and pedestrian traffic demand $(25 \mathrm{~s}$ green light time and $5 \mathrm{~m}$ crosswalk width).

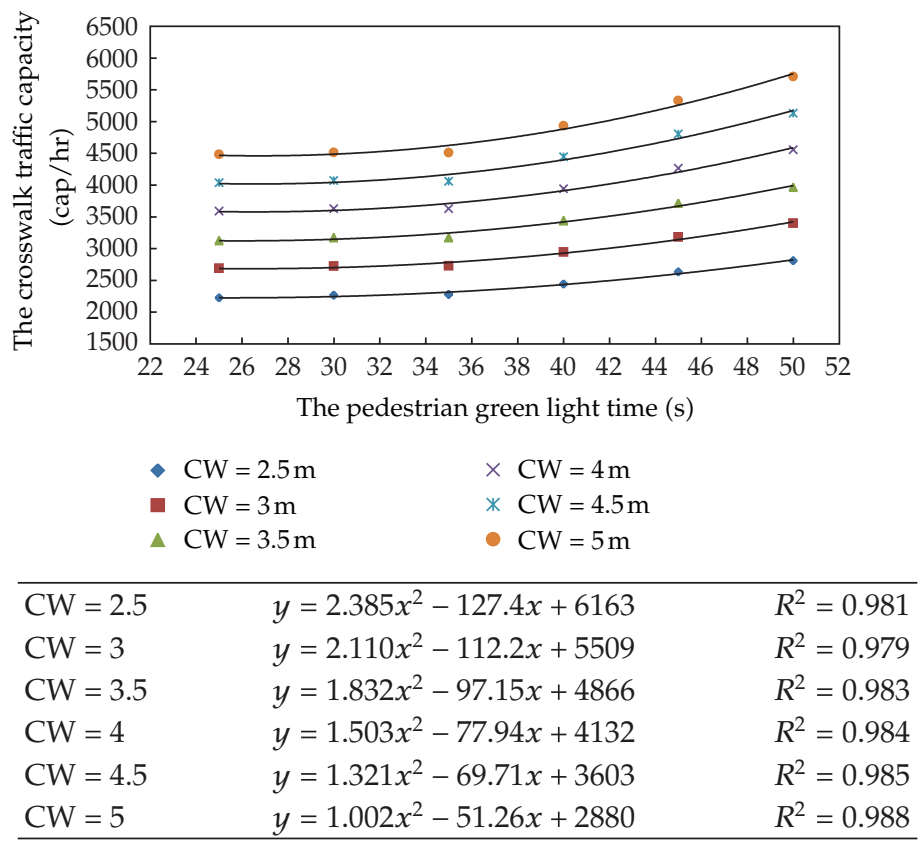

Figure 4: Relationship between pedestrian traffic capacity of crosswalk and green length. 


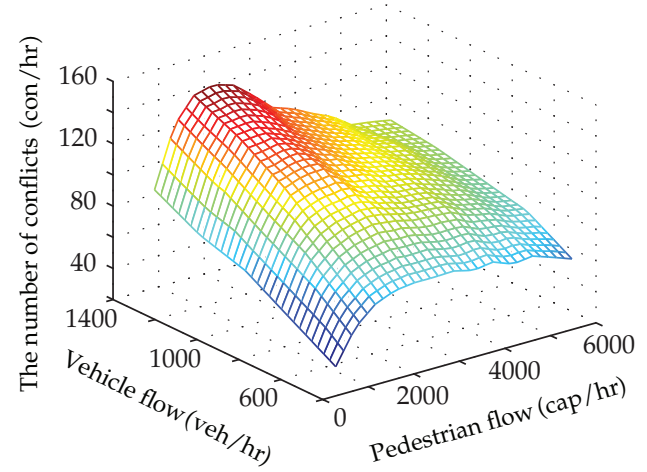

(a) $50 \mathrm{~s}$ green light and $3 \mathrm{~m}$ crosswalk width

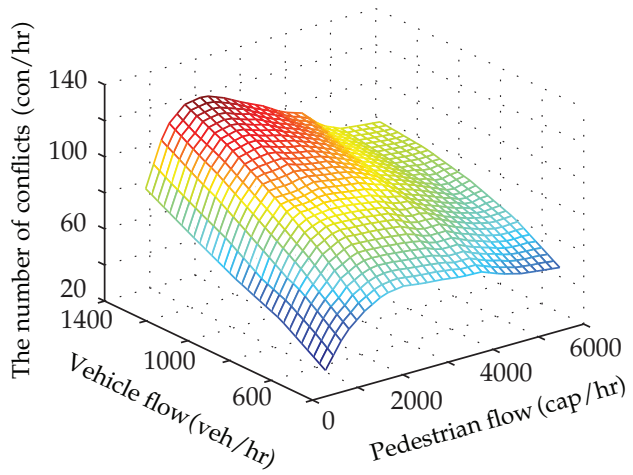

(c) $40 \mathrm{~s}$ green light and $3 \mathrm{~m}$ crosswalk width

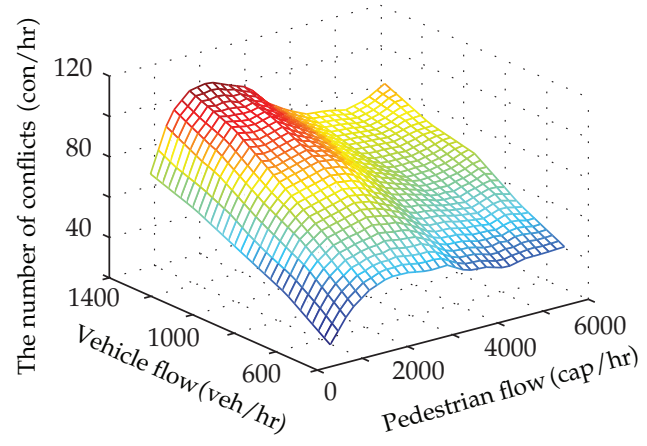

(e) $30 \mathrm{~s}$ green light and $3 \mathrm{~m}$ crosswalk width

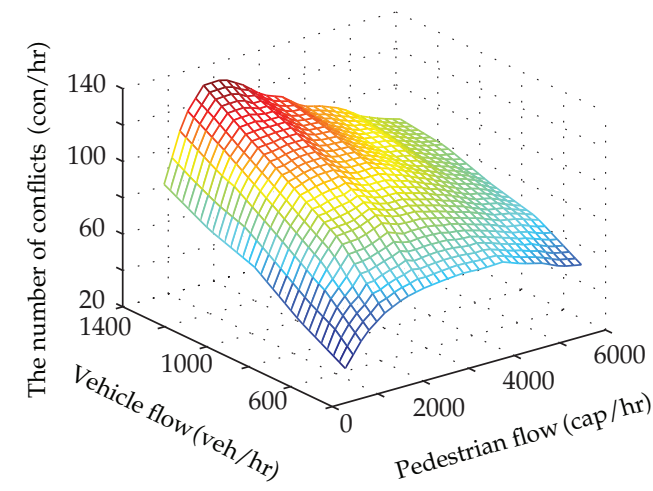

(b) $45 \mathrm{~s}$ green light and $3 \mathrm{~m}$ crosswalk width

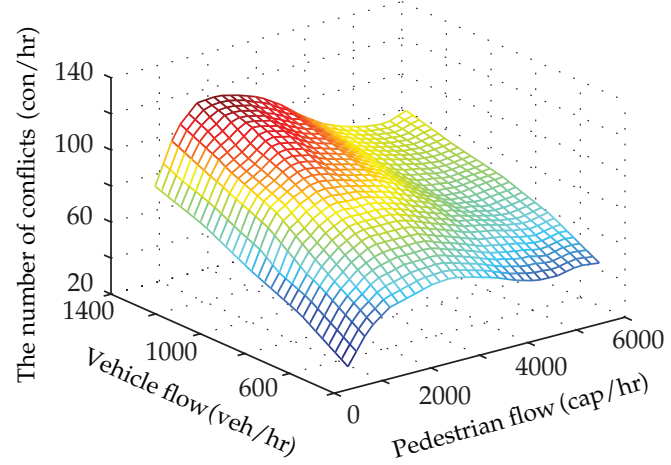

(d) $35 \mathrm{~s}$ green light and $3 \mathrm{~m}$ crosswalk width

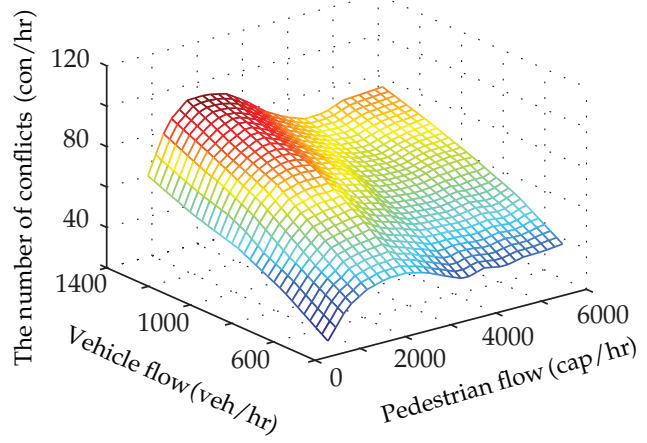

(f) $25 \mathrm{~s}$ green light and $3 \mathrm{~m}$ crosswalk width

Figure 5: Relationship among pedestrian flow, vehicle flow, and the number of conflict events between pedestrians and vehicles for different types of pedestrian green lengths.

\subsection{Conflict Events between Pedestrians and Vehicles}

Figure 5 illustrates the relationship among pedestrian flow, vehicle flow, and the number of conflict events between pedestrians and vehicles for different types of pedestrian green lengths when the crosswalk width is $3 \mathrm{~m}$. It indicates that the number of conflict events is monotonously increasing with the vehicle flow. However, it is increasing with the pedestrian flow first, and once it reaches the maximum value, it then decreases. This interesting 
finding can be explained that when the pedestrian density in crosswalk is low, the increase of the pedestrian flow engenders more conflicts. As the pedestrian flow and density increase further, it leads to the smaller possibility that they were interrupted by vehicles while crossing, so the downtrend appears. Therefore, the maximum number of conflicts between pedestrians and vehicles exists corresponding to a certain level of pedestrian flow. Furthermore, the result shows that the number of conflict events decreases with decrease of the green length of pedestrian signal. Intuitively, as the green length increases, more pedestrians can cross the intersection and have chances to encounter conflicts with vehicles during a signal cycle. However, crosswalk width has no significant effect on the number of conflict events, as shown in Figure 6.

According to the four areas mentioned early, the relationship of the conflict events generated in each area is shown in Figure 7 (the vehicle flow is 648 per hour). Among them Area 1 has the most conflict events, followed by Area 4, Area 3, and Area 2 in order. As the right-turning vehicles are not restricted by the red light at the intersection, more conflicts occur in Area 1 and Area 4. It implies that Area 1 and Area 4 would be prone to be safety black spots of vehicle-pedestrian crashes in intersection crosswalks. This simulation-based conclusion is consistent with many previous research results [47-49]. For the straight-moving or left-turning vehicles from inside of intersection in Area 2, they need a longer period of time to arrive at the crosswalk after the pedestrian light turns red. So, there are fewer conflicts between red-light running pedestrians and vehicles in Area 2 compared to Area 3.

\subsection{Pedestrian Conflict Delay}

Figure 8 shows the trend of total pedestrian conflict delay with vehicle flow and pedestrian flow at different types of crosswalk width $(2.5 \mathrm{~m}, 3 \mathrm{~m}, 3.5 \mathrm{~m}, 4 \mathrm{~m}, 4.5 \mathrm{~m}$, and $5 \mathrm{~m})$ when the green length is $35 \mathrm{~s}$. It is found that the total pedestrian conflict delay is increasing as either vehicle flow or pedestrian flow increases; however, crosswalk width does not have a significant effect on pedestrian conflict delay. Figure 9 shows the effect of different types of signal timing $(50 \mathrm{~s}, 45 \mathrm{~s}, 40 \mathrm{~s}, 35 \mathrm{~s}, 30 \mathrm{~s}$, and $25 \mathrm{~s}$ green length) on the total pedestrian conflict delay when the crosswalk width is definite $(3 \mathrm{~m})$ and the pedestrian flow is 4320 per hour. An interesting phenomenon is that when the pedestrian green light time is longer than 40 seconds, the total pedestrian conflict delay decreases as the green light time decreases; however, when the green light time is less than 40 seconds, the total pedestrian conflict delay increases significantly. The reason can be explained as follows. When the pedestrian green light time is relatively long, the conflicts between right-turning vehicles and pedestrians (conflicts in Area 1 and Area 4) decrease as the green length decreases, which are the main source of pedestrian conflict delay since the right turn on red is not restricted at the intersection. Thus, the conflict delay decreases. As the green light time decreases continuously till $35 \mathrm{~s}$, quite a number of pedestrians who cannot complete crossing during green light are stagnated in the middle of the crosswalk when the red light begins. As they have to continue to cross the intersection, more conflicts happen between them and vehicles in Area 2 and Area 3. So, the total conflict delay increases abruptly when the green light time is short enough.

Additionally, during pedestrian crossing, there is not only conflict delay but also redlight stop delay for pedestrians and both of them constitute the pedestrian crossing delay. The pedestrian red light delay means the time during which pedestrians stop at intersections waiting for green signal. Obviously the delay is inexistent for pedestrians running the red light illegally. Table 1 lists a comparison between pedestrian conflict delay and red light delay 


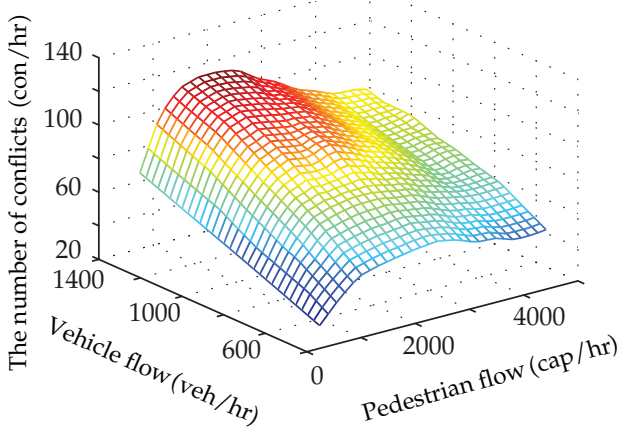

(a) $35 \mathrm{~s}$ green light and $2.5 \mathrm{~m}$ crosswalk width

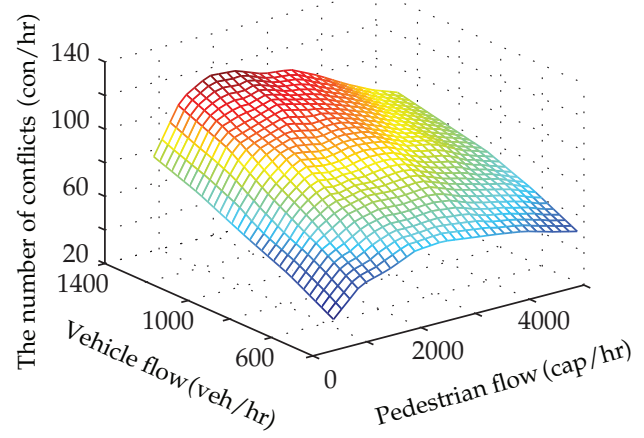

(c) $35 \mathrm{~s}$ green light and $3.5 \mathrm{~m}$ crosswalk width

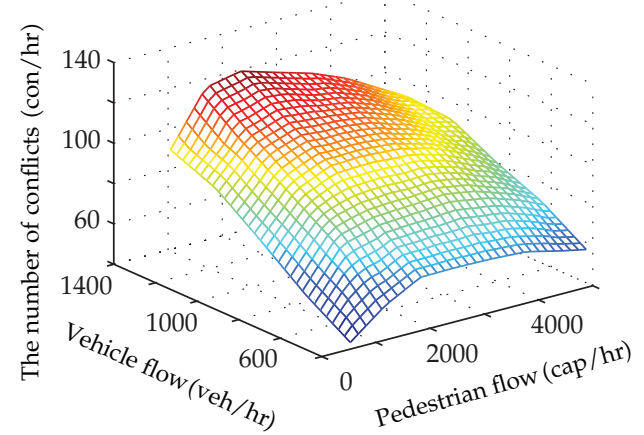

(e) $35 \mathrm{~s}$ green light and $4.5 \mathrm{~m}$ crosswalk width

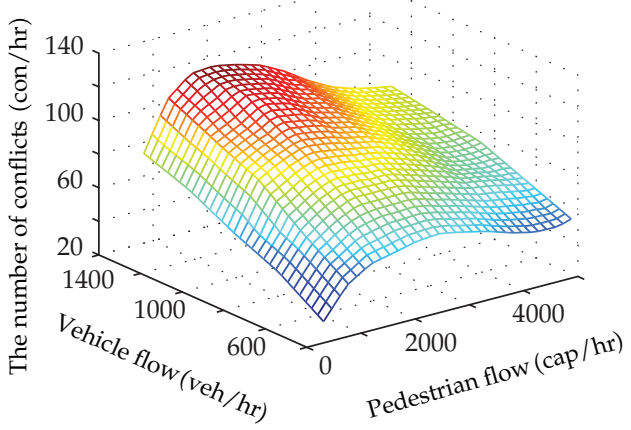

(b) $35 \mathrm{~s}$ green light and $3 \mathrm{~m}$ crosswalk width

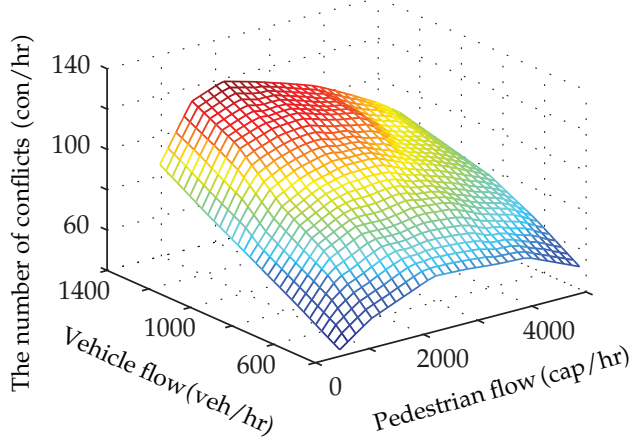

(d) $35 \mathrm{~s}$ green light and $4 \mathrm{~m}$ crosswalk width

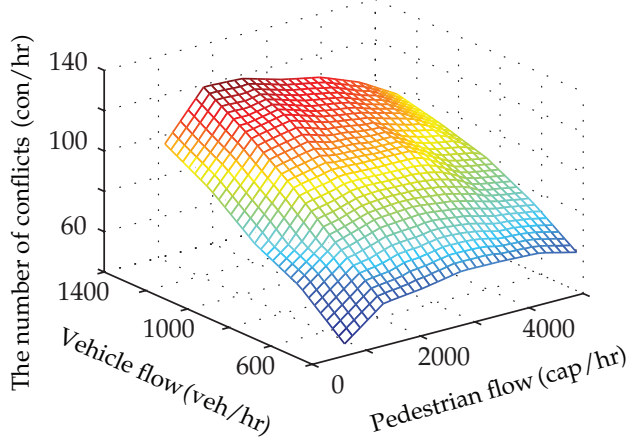

(f) $35 \mathrm{~s}$ green light and $5 \mathrm{~m}$ crosswalk width

Figure 6: Relationship among pedestrian flow, vehicle flow, and the number of conflict events between pedestrians and vehicles for different types of crosswalk widths.

per capita when the vehicle arrival rate $\left(\lambda_{v}\right)$ is 0.06 and the pedestrian arrival rate $\left(\lambda_{p}\right)$ ranges from 0.07 to 0.13 . An important finding is that the conflict delay constitutes quite a large ratio in the total pedestrian crossing delay, which can even reach $41 \%$ maximally. Thus the conflict delay's influence on the pedestrian crossing efficiency and safety cannot be ignored.

\section{Conclusion}

Few researchers paid attention to the pedestrian and vehicle conflicts with a microscopic simulation method. In this paper, the pedestrian and vehicle behaviors and the conflicts between 


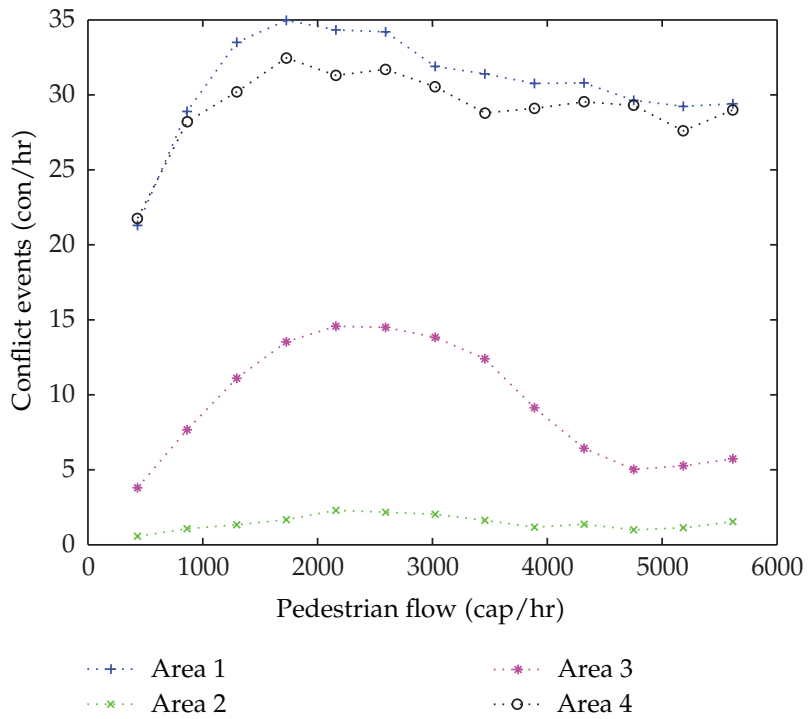

Figure 7: The conflict events in four areas with green light time $35 \mathrm{~s}$ and crosswalk width $3 \mathrm{~m}$.

Table 1: The pedestrian conflict delay and red light delay.

\begin{tabular}{lcccccccccc}
\hline \multirow{2}{*}{ Green light time (s) } & \multirow{2}{*}{ Delay type } & \multirow{2}{*}{$\lambda_{v}$} & & \multicolumn{7}{c}{ Pedestrian delay (s/cap) } \\
& & & & \multicolumn{7}{c}{$\lambda_{p}$} \\
& & & 0.07 & 0.08 & 0.09 & 0.10 & 0.11 & 0.12 & 0.13 \\
\hline \multirow{2}{*}{50} & RLD & 0.06 & 18.76 & 18.58 & 18.33 & 18.09 & 18.14 & 17.87 & 17.67 \\
& CD & 0.06 & 3.43 & 3.96 & 4.37 & 4.75 & 5.69 & 6.09 & 7.03 \\
\hline \multirow{2}{*}{45} & RLD & 0.06 & 21.24 & 21.01 & 20.75 & 20.45 & 20.30 & 20.05 & 19.80 \\
& CD & 0.06 & 3.49 & 3.86 & 4.31 & 4.58 & 4.92 & 5.70 & 6.22 \\
\hline \multirow{2}{*}{40} & RLD & 0.06 & 23.56 & 23.22 & 23.00 & 22.83 & 22.59 & 22.38 & 22.20 \\
& CD & 0.06 & 2.99 & 2.93 & 3.30 & 3.49 & 3.95 & 4.32 & 4.83 \\
\hline \multirow{2}{*}{35} & RLD & 0.06 & 25.70 & 25.73 & 25.44 & 25.16 & 24.87 & 24.52 & 24.43 \\
& CD & 0.06 & 7.33 & 10.54 & 11.27 & 14.75 & 15.26 & 16.61 & 17.03 \\
\hline \multirow{2}{*}{25} & RLD & 0.06 & 28.13 & 27.91 & 27.75 & 27.38 & 27.16 & 27.12 & 26.82 \\
& CD & 0.06 & 8.30 & 10.15 & 12.55 & 13.26 & 15.45 & 15.54 & 15.53 \\
\hline
\end{tabular}

RLD represents red light delay.

$\mathrm{CD}$ represents conflict delay.

them were simulated through cellular automata evolution rules. Using the simulation model, we investigated the effects of pedestrian signal timing and crosswalk width on the crosswalk capacity, the number of traffic conflicts between pedestrians and vehicles, and pedestrian delay due to the conflicts.

The simulation results showed that the maximum pedestrian traffic capacity on the crosswalk has a strong quadratic mathematical relationship with the signal timing for different crosswalk widths. The number of conflict events between pedestrians and vehicles declines with decrease of vehicle flow and pedestrian signal green length, but is not significantly influenced by crosswalk width. An interesting finding is that, given a fixed 


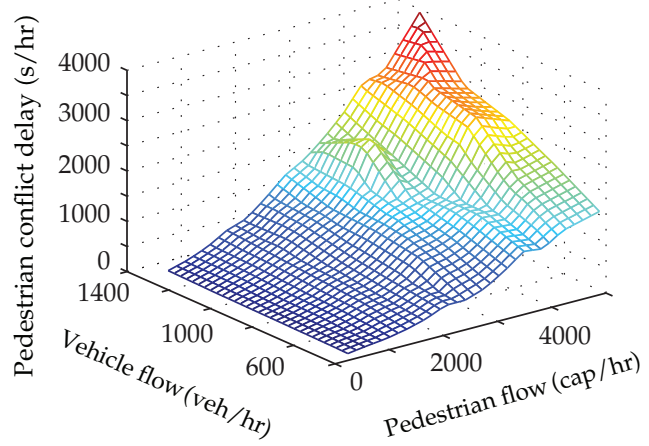

(a) $35 \mathrm{~s}$ green light and $2.5 \mathrm{~m}$ crosswalk width

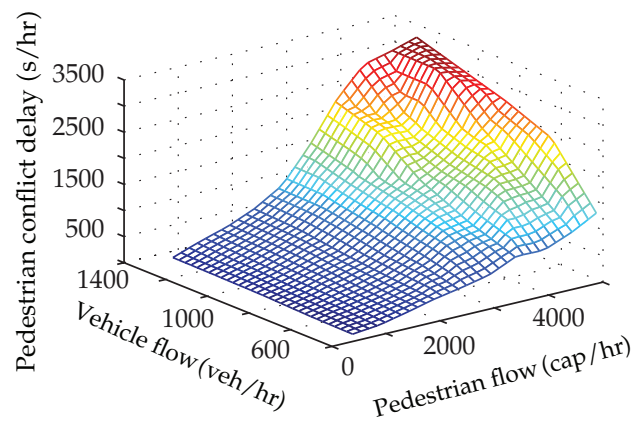

(c) $35 \mathrm{~s}$ green light and $3.5 \mathrm{~m}$ crosswalk width

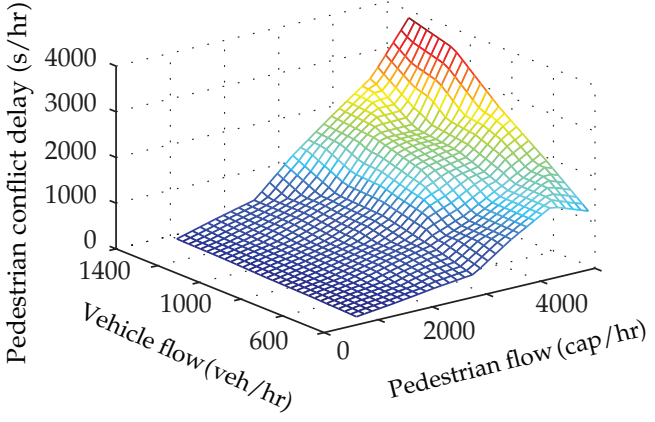

(e) $35 \mathrm{~s}$ green light and $4.5 \mathrm{~m}$ crosswalk width

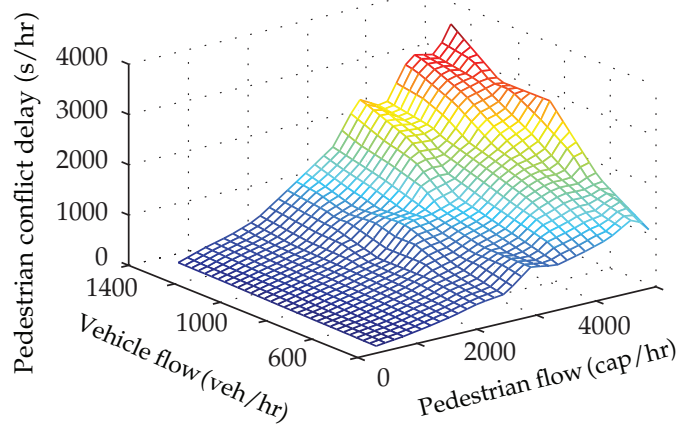

(b) $35 \mathrm{~s}$ green light and $3 \mathrm{~m}$ crosswalk width

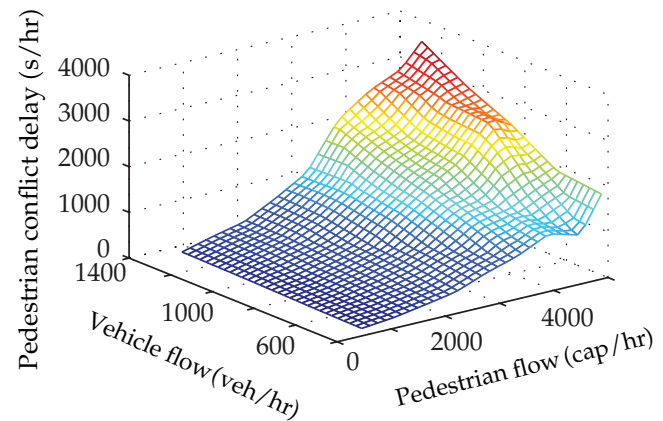

(d) $35 \mathrm{~s}$ green light and $4 \mathrm{~m}$ crosswalk width

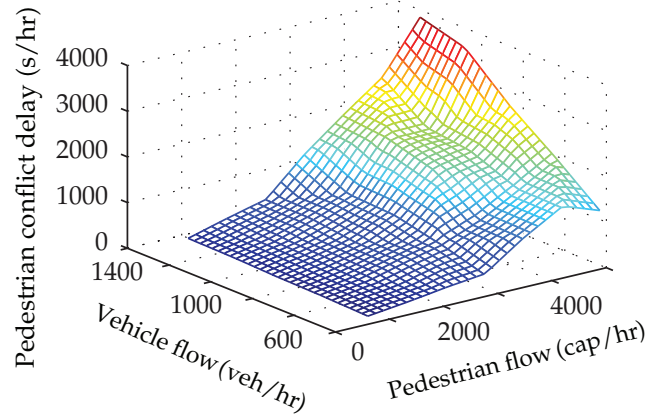

(f) $35 \mathrm{~s}$ green light and $5 \mathrm{~m}$ crosswalk width

Figure 8: Relationship among pedestrian flow, vehicle flow, and pedestrian conflict delay for different types of crosswalk widths.

vehicle flow, there exists the maximum number of conflicts between pedestrians and vehicles corresponding to a certain level of pedestrian flow. Furthermore, it was found that the pedestrian conflict delay is increasing as either vehicle flow or pedestrian flow increases. However, crosswalk width has no significant influence on the conflict delay. An interesting observation is that as the pedestrian signal green length increases, there is an abrupt decrease in pedestrian conflict delay. According to the comparison between pedestrian red-light stop delay and conflict delay, the latter constitutes quite a large portion in the total pedestrian crossing delay, which can be up to $41 \%$ at maximum. Therefore, the conflicts between 


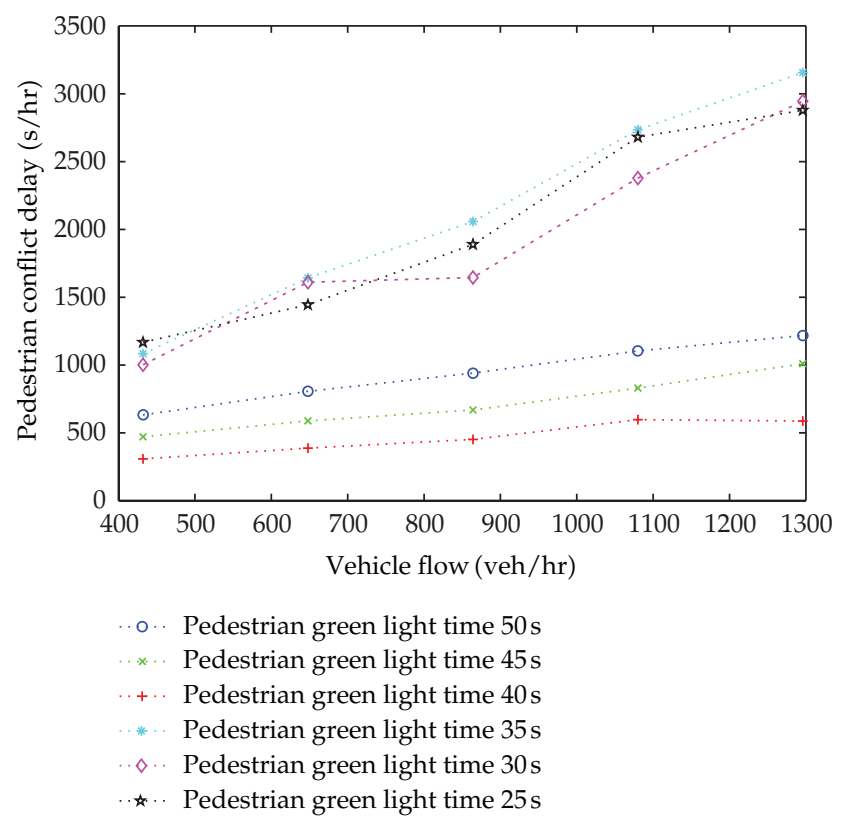

Figure 9: The relationship between pedestrian conflict delay and green light time.

pedestrians and vehicles at intersections not only induce traffic safety problems but also contribute to the efficiency decline of pedestrian traffic operation to a substantial degree.

Previous studies have investigated dynamics characteristics of road traffic flow under the effect of traffic light with cellular automata method from a micropoint of view. It was found that the global throughput of the network strongly depends on the cycle times and strong oscillations in the global flow are found in dependence of the cycle times for both low and high densities [50]; Biham et al. found a sharp jamming transition that separates between the low-density dynamical phase in which all cars move at maximal speed and the high-density jammed phase in which they are all stuck [51]; Chowdhury and Schadschneider investigated the time dependence of the average speeds of the cars in the "free-flowing" phase as well as the dependence of flux and jamming on the time period of the signals [52]. However, in this paper we explored the effect of design factors on the crosswalk capacity, the conflict event, and related delay, which illustrates that the cellular automata application can be used as a practical tool to search for better engineering solutions to enhance intersection layout design and signal timing.

In summary, the cellular automata simulation for interaction between pedestrian and vehicle behaviors in this study displays complex characteristics of mixed traffic flow and provides a useful method to analyze pedestrian operation in crosswalks at intersections.

\section{Acknowledgments}

This work is financially supported by Chinese National 973 Project (2012CB725403), National Natural Science Foundation (71171014), Ph.D. Programs Foundation of Ministry of Education of China (20110009110013), Youth Thousand Talents Program (T12FR00010), the State Key Laboratory of Rail Traffic Control and Safety (RCS2011ZT007), and Program for New Century Excellent Talents in University (NCET-11-0570). 


\section{References}

[1] D. Y. Zhang, Road Traffic Safety Management Evaluation System, People's Traffic Press, Beijing, China, 2005.

[2] S. R. Perkins and J. I. Harris, Traffic Conflict Characteristics: Accident Potential at Intersections, Highway Research Record No. 225, Traffic Safety and Accident Research, 1968, 6 Reports.

[3] F. Amundson and C. Hyden, in Proceedings of the 1st Workshop on TrafficConflicts, Institute of Economics, Oslo, Norway, 1977.

[4] E. Hauer, "Traffic conflicts and exposure," Accident Analysis and Prevention, vol. 14, no. 5, pp. 359-364, 1982.

[5] E. Hauer, Observational before-after Studies in Road Safety, Pergamon, Oxford, UK, 1997.

[6] P. Cooper, "Experience with traffic conflicts in canada with emphasis on post encroachment time techniques," in International Study of Traffic Conflict Techniques, E. Asmussen, Ed., pp. 75-96, Springer, 1984.

[7] D. Migletz, W. Glauz, and K. Bauer, "Relationships between traffic conflicts and accidents," Report FHWA/RD-84/042, Federal Highway Administration, Washington, DC, USA, 1985.

[8] M. Ben-Akiva and S. R. Lerman, Discrete Choice Analysis: Theory and Application to Travel Demand, The MIT Press, Cambridge, Mass, USA, 1985.

[9] J. M. Cassidy, S. M. Madanat, M. Wang, and F. Yang, “Unsignalized intersection capacity and level of service: revisiting critical gap," Transportation Research Record 1484, TRB, National Research Council, Washington, DC, USA, 1995.

[10] H. Mahmassani and Y. Sheffi, "Using gap sequences to estimate gap acceptance functions," Transportation Research B, vol. 15, no. 3, pp. 143-148, 1981.

[11] L. P. Gao, M. J. Liu, and J. Feng, "Delay modeling of Ped-Veh system based on pedestrian crossing at signalized intersection," in Proceedings of the 8th International Conference on Traffic and Transportation Studies Changsha (ICTTS '12), Beijing, China, 2012.

[12] J. Y. S. Lee and W. H. K. Lam, "Simulating pedestrian movements at signalized crosswalks in Hong Kong," Transportation Research Part A, vol. 42, no. 10, pp. 1314-1325, 2008.

[13] W. K. M. Alhajyaseen, M. Asano, and H. Nakamura, "Estimation of left-turning vehicle maneuvers for the assessment of pedestrian safety at intersections," IATSS Research, vol. 36, no. 1, pp. 66-74, 2012.

[14] H. W. Kruysse, "The subjective evaluation of traffic conflicts based on an internal concept of dangerousness," Accident Analysis and Prevention, vol. 23, no. 1, pp. 53-65, 1991.

[15] A.-W. Zheng, J.-Z. Guo, and Z.-M. Niu, "Analysis of traffic conflicting and the influence factors," Journal of Wuhan University of Science and Technology, vol. 25, no. 4, p. 367, 2002 (Chinese).

[16] G. Q. Zhang, "Determination of the severity of traffic conflict at highway intersections," Journal of Hefei University of Technology, vol. 31, no. 5, pp. 683-686, 2008 (Chinese).

[17] G. Tiwari, D. Mohan, and J. Fazio, "Conflict analysis for prediction of fatal crash locations in mixed traffic streams," Accident Analysis and Prevention, vol. 30, no. 2, pp. 207-215, 1998.

[18] Q. Fang and Z. Wu, "Prediction of traffic conflict at intersection based on gray control theory," Communications Standardization, vol. 6, pp. 174-177, 2008 (Chinese).

[19] W. Cheng and G. Y. Wang, "Prediction of traffic conflicts at intersection based on adaptive neuralfuzzy inference system," Journal of Highway and Transportation Research and Development, vol. 22, no. 7, pp. 115-117, 2005 (Chinese).

[20] T. M. Mohammed, Development of a conflict rate prediction model at non-signalized intersections [Ph.D. thesis], University of South Florida, 2003.

[21] E. Hauer and P. Garder, "Research into the validity of the traffic conflicts technique," Accident Analysis and Prevention, vol. 18, no. 6, pp. 471-481, 1986.

[22] T. Zhu, Y. Bai, and X. G. Yang, "Failure analysis on safety assessment method for traffic conflicts at level crossing and its improvement," China Safety Science Journal, vol. 18, no. 2, pp. 157-161, 2008 (Chinese).

[23] P. Gårder, "Pedestrian safety at traffic signals: a study carried out with the help of a traffic conflicts technique," Accident Analysis and Prevention, vol. 21, no. 5, pp. 435-444, 1989.

[24] D. Helbing, R. Jiang, and M. Treiber, "Analytical investigation of oscillations in intersecting flows of pedestrian and vehicle traffic," Physical Review E, vol. 72, no. 4, Article ID 046130, 2005.

[25] P. G. Gipps and B. Marksjö, "A micro-simulation model for pedestrian flows," Mathematics and Computers in Simulation, vol. 27, no. 2-3, pp. 95-105, 1985.

[26] K. Nagel and M. Schreckenberg, "A cellular automaton model for freeway traffic," Journal of Physique, vol. 2, no. 12, pp. 2221-2228, 1992. 
[27] K. Nagel and S. Rasmussen, "Traffic at the edge of chaos," in Proceedings of the 4th International Workshop on the Synthesis and Simulation of Living Systems Artificial Life IV, pp. 222-225, 1994.

[28] M. Rickert, K. Nagel, M. Schreckenberg, and A. Latour, "Two-lane traffic simulations using cellular automata," Los Alamos Unclassified Report 95:4367, Los Alamos National Laboratory, Los Alamos, NM, USA, 1996.

[29] M. Rickert, K. Nagel, M. Schreckenberg, and A. Latour, "Two-lane traffic simulations using cellular automata," Physica A, vol. 231, p. 534, 1995.

[30] V. J. Blue, M. J. Embrechts, and J. L. Adler, "Cellular automata modeling of pedestrian movements," in Proceedings of the IEEE International Conference on Systems, Man, and Cybernetics, vol. 3, pp. 2320-2323, October 1997.

[31] S. Maerivoet and B. De Moor, "Cellular automata models of road traffic," Physics Reports, vol. 419, no. 1, pp. 1-64, 2005.

[32] V. J. Blue and J. L. Adler, "Cellular automata microsimulation for modeling bi-directional pedestrian walkways," Transportation Research B, vol. 35, no. 3, pp. 293-312, 2001.

[33] K. Yamamoto, S. Kokubo, and K. Nishinari, "Simulation for pedestrian dynamics by real-coded cellular automata (RCA)," Physica A, vol. 379, no. 2, pp. 654-660, 2007.

[34] C. Burstedde, K. Klauck, A. Schadschneider, and J. Zittartz, "Simulation of pedestrian dynamics using a two-dimensional cellular automaton," Physica A, vol. 295, no. 3-4, pp. 507-525, 2001.

[35] W. G. Weng, T. Chen, H. Y. Yuan, and W. C. Fan, "Cellular automaton simulation of pedestrian counter flow with different walk velocities," Physical Review E, vol. 74, no. 3, Article ID 036102, 2006.

[36] D. Chowdhury, L. Santen, and A. Schadschneider, "Statistical physics of vehicular traffic and some related systems," Physics Reports, vol. 329, no. 4-6, pp. 199-329, 2000.

[37] B. Chopard and A. Masselot, "Cellular automata and lattice Boltzmann methods: a new approach to computational fluid dynamics and particle transport," Future Generation Computer Systems, vol. 16, no. 2, pp. 249-257, 1999.

[38] E. F. Codd, Cellular Automata, Academic Press, New York, NY, USA, 1968.

[39] M. Gardner, "Mathematical games-the fantastic combinations of John Conway's new solitaire game, 'Life'," Scientific American, vol. 223, pp. 120-123, 1970.

[40] M. Cremer and J. Ludwig, "A fast simulation model for traffic flow on the basis of boolean operations," Mathematics and Computers in Simulation, vol. 28, no. 4, pp. 297-303, 1986.

[41] D. Helbing and T. Vicsek, "Optimal self-organization," New Journal of Physics, vol. 1, pp. 13.1-13.17, 1999.

[42] I. Karafyllidis and A. Thanailakis, "A model for predicting forest fire spreading using cellular automata," Ecological Modelling, vol. 99, no. 1, pp. 87-97, 1997.

[43] K. Nagel and E. Raschke, "Self-organizing criticality in cloud formation?" Physica A, vol. 182, no. 4, pp. 519-531, 1992.

[44] S. Gobron and N. Chiba, "Crack pattern simulation based on 3D surface cellular automata," Visual Computer, vol. 17, no. 5, pp. 287-309, 2001.

[45] K. Nishinari, D. Chowdhury, and A. Schadschneider, "Cluster formation and anomalous fundamental diagram in an ant-trail model," Physical Review E, vol. 67, no. 3, Article ID 036120, 2003.

[46] L. Yang, W. Fang, R. Huang, and Z. Deng, “Occupant evacuation model based on cellular automata in fire," Chinese Science Bulletin, vol. 47, no. 17, pp. 1484-1488, 2002.

[47] D. F. Preusser, W. A. Leaf, K. B. DeBartolo, R. D. Blomberg, and M. M. Levy, "The effect of rightturn-on-red on pedestrian and bicyclist accidents," Journal of Safety Research, vol. 13, no. 2, pp. 45-55, 1982.

[48] J. Autey, T. Sayed, and M. H. Zaki, "Safety evaluation of right-turn smart channels using automated traffic conflict analysis," Accident Analysis \& Prevention, vol. 45, pp. 120-130, 2012.

[49] Y. Ni and K. P. Li, "Dealing with conflict between pedestrian and right turning vehicle in signalized intersection," Computer and Communications, vol. 25, no. 1, pp. 22-26, 2007 (Chinese).

[50] E. Brockfeld, R. Barlovic, A. Schadschneider, and M. Schreckenberg, "Optimizing traffic lights in a cellular automaton model for city traffic," Physical Review E, vol. 64, no. 5, Article ID 056132, 2001.

[51] O. Biham, A. A. Middleton, and D. Levine, "Self-organization and a dynamical transition in trafficflow models," Physical Review A, vol. 46, no. 10, pp. R6124-R6127, 1992.

[52] D. Chowdhury and A. Schadschneider, "Self-organization of traffic jams in cities: effects of stochastic dynamics and signal periods," Physical Review E, vol. 59, no. 2, pp. R1311-R1314, 1999. 


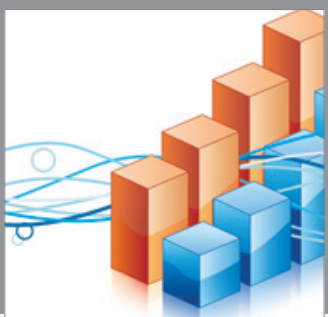

Advances in

Operations Research

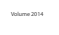

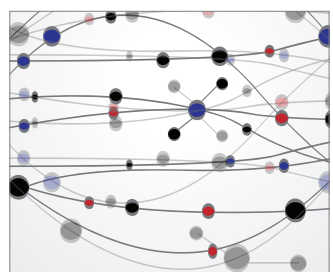

\section{The Scientific} World Journal
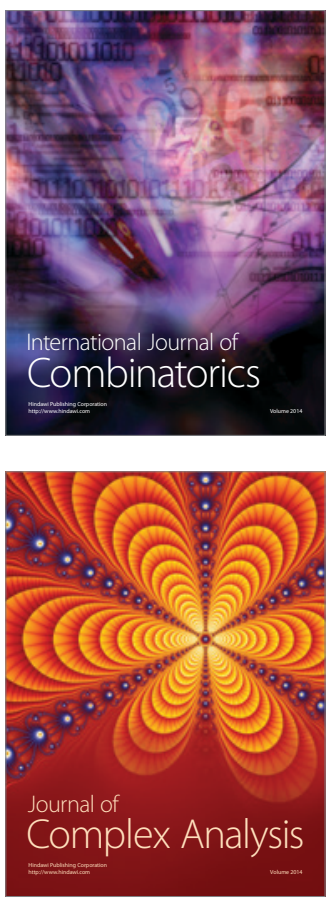

International Journal of

Mathematics and

Mathematical

Sciences
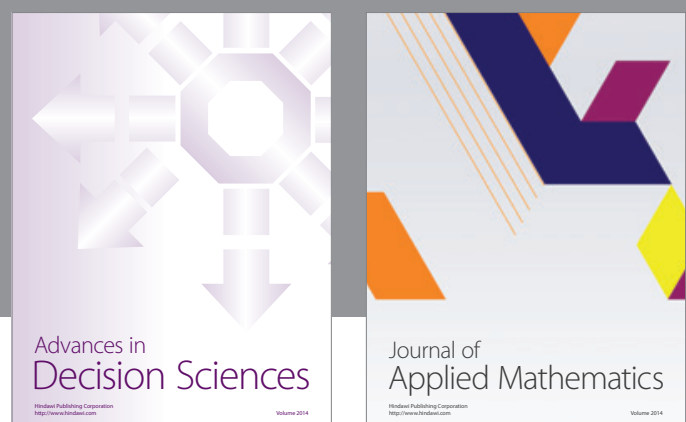

Journal of

Applied Mathematics
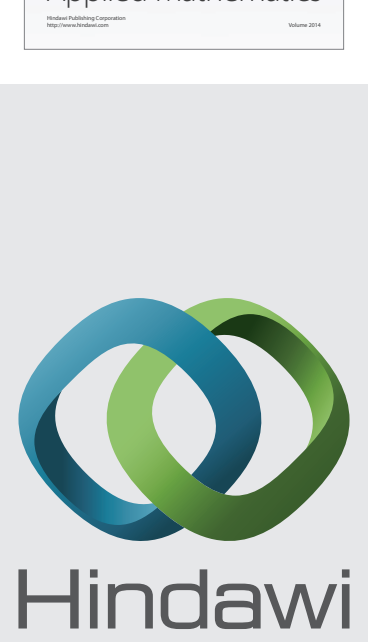

Submit your manuscripts at http://www.hindawi.com
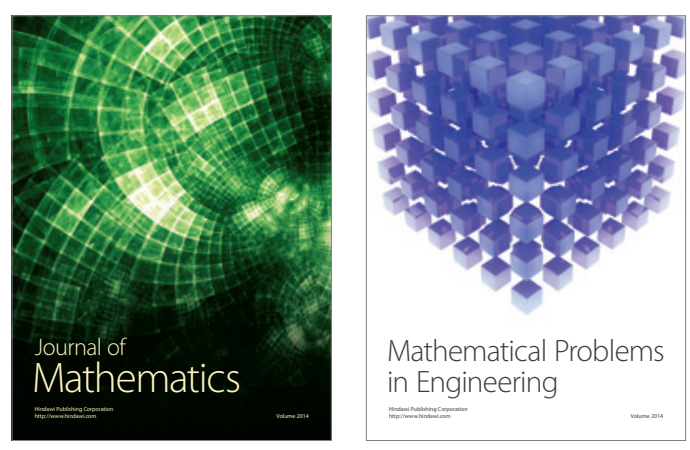

Mathematical Problems in Engineering
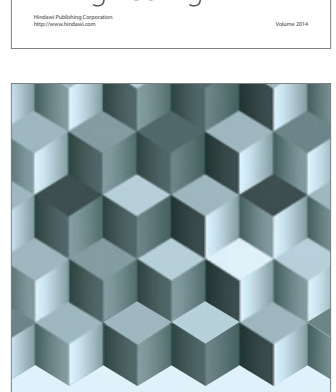

Journal of

Function Spaces
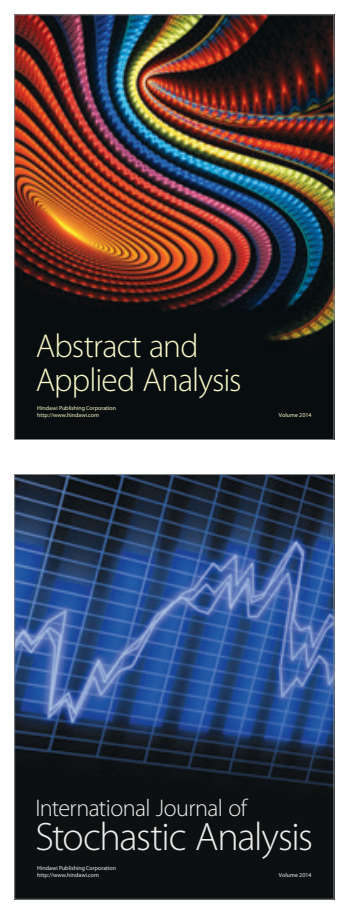

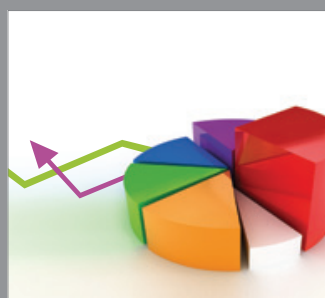

ournal of

Probability and Statistics

Promensencen
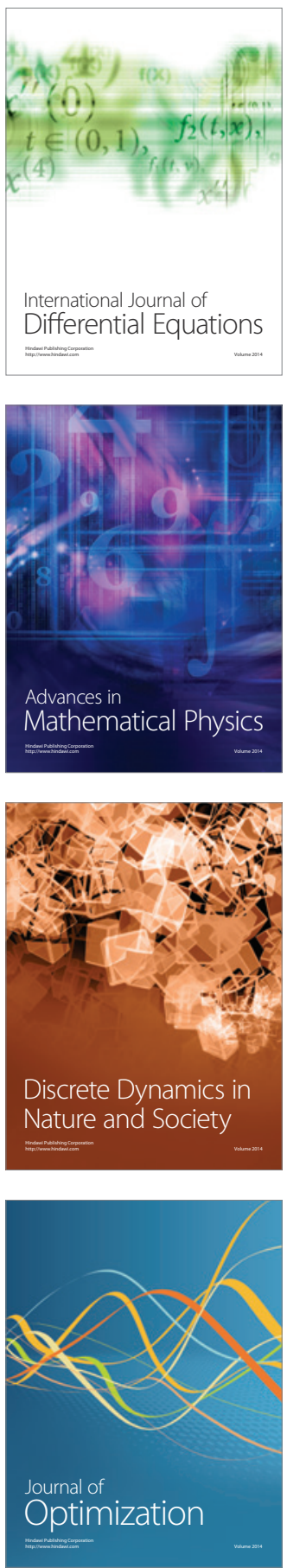